\title{
Simulación digital en MOOdLE PARA LA ENSEÑANZA DEL DESARROLlo DEL VOCABULARIO INFANTIL
}

\author{
Alba Selene Hernández Viveros, Esperanza Guarneros Reyes y Argenis Josué Espinoza Zepeda \\ FES Iztacala UNAM \\ México
}

\begin{abstract}
RESUMEN
Este trabajo está enfocado al diseño y elaboración de un simulador digital en Moodle para la enseñanza del desarrollo del vocabulario infantil como una herramienta interactiva de ensayo al alcance de los estudiantes del SUAyED Iztacala de la carrera de Psicología; la metodología del diseño fue simulación por ramificación, pues permite al estudiante tomar decisiones acerca de las situaciones que se le presentan; el desarrollo consistió en una capacitación de los software a utilizar, para después diagramar la situación simulada, trasladarla a un guión y hacer el montaje en la plataforma de Moodle; se hizo una evaluación por pares y con expertos en el tema para realizar las modificaciones pertinentes, resultando un espacio de prácticas virtuales para el estudiante; se concluyó que con el uso de herramientas libres, como audio, videos y configuraciones en una plataforma educativa se puede crear interacción mediante un simulador digital.
\end{abstract}

\section{Palabras Clave:}

simulación digital, simulaciones remificadas, toma de decisiones, Moodle

\section{Digital simulation in MoOdle to teach the deVelopment of CHILDREN'S VOCABULARY}

\begin{abstract}
This work is focused on the design and development of a Digital Simulator in Moodle for the Teaching of the Development of Children's Vocabulary, as an interactive essay tool, which may be available to students of SUAyED Iztacala of the Psychology career, the method used was branching simulation, which allows the student to make decisions about the presented situations; the development consisted of training about the softwares to be used, to then diagram the simulated situation, transfer it to a script and carry out the assembly on the Moodle platform; Afterwards, a peer and expert evaluation of the subject was made, to finally make the pertinent modifications, resulting in a space of virtual practices for the student so it is concluded that, with the use of free tools, such as audio, videos and configurations within an educational platform, interaction can be created through a Digital Simulator.
\end{abstract}

\section{Keywords:}

digital simulation, branching simulation, decision, vocabulary, Moodle.

Bitácora del Artículo:

| Recibido: 21 de noviembre de 2019 | Aceptado: 8 de junio de 2020 | Publicado en línea: Julio-Diciembre de 2020 | 
Innovación y desarrollo tecnológico | Simulación digital en Moodle ... Hernández-Viveros, Guarneros-Reyes \& Espinoza-Zepeda

\title{
Autoría y Derechos de Propiedad Intelectual
}

\section{SiMULACIÓN DIGITAL EN MOODLE PARA LA ENSEÑANZA DEL DESARROLLO DEL VOCABULARIO INFANTIL}

\author{
Alba Selene Hernández Viveros, Esperanza Guarneros Reyes y Argenis Josué Espinoza Zepeda \\ FES Iztacala UNAM \\ México
}

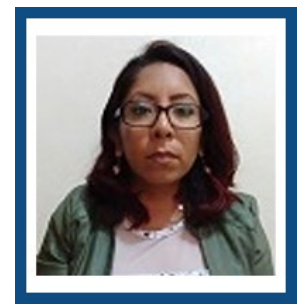

Alba Selene Hernández Viveros FES Iztacala UNAM Correo: psic.albahernandez@gmail.com

Licenciada en Psicología por la FES Iztacala. Tutora en el Programa Institucional de Tutorías para estudiantes de nuevo ingreso del SUAyED.Actualmente ofrece psicoterapia multicultural con perspectiva de género en la UMF 31 de Iztapalapa. En la FESI proporcionó atención a crisis y emergencias psicológicas a jóvenes con riesgo suicida.

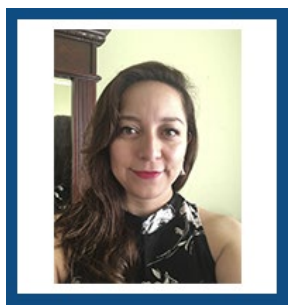

Esperanza Guarneros Reyes

FES Iztacala UNAM

Correo: esperanzagr@gmail.com

Doctora en Psicología por la Universidad Nacional Autónoma de México UNAM, es jefa del Laboratorio Digital de Desarrollo Infantil LDDI, es profesora de carrera Titular A de tiempo completo definitiva de la carrera de psicología en línea, además de impartir clase en la licenciatura de psicología presencial y maestría en la UNAM, FES Iztacala, México; investiga sobre habilidades lingüísticas en preescolares y experta en ambientes virtuales de aprendizaje.

\section{CONTRIBUCIÓN DE LOS AUTORES}

Alba Selene Hernández Viveros, fue colaboradora del Laboratorio Digital de Desarrollo Infantil en el proyecto PAPIME PE307717, en este proyecto realizó el simulador en Moodle sobre el desarrollo del vocabulario de niños preescolares y concluyó su manuscrito de titulación, participó en la escritura del artículo. | Esperanza Guarneros Reyes, formuló la idea original del simulador digital por ramificación en Moodle para el desarrollo del vocabulario y dirigió su desarrollo como responsable del proyecto UNAM-PAPIME PE307717, así como la escritura del artículo. | Argenis Josué Espinoza Zepeda, capacitó a los participantes, como el caso de Alba, en el uso de herramientas de Moodle para realizar simuladores digitales y realizó los esquemas para diagramar los simuladores.

\section{AGRADECIMIENTOS}

Investigación realizada gracias al Programa UNAM-PAPIIT IN306218 y PAPIME PE307717

\section{DATOS DE Filiación de los AUtORES}

Facultad de Estudios Superiores Iztacala UNAM

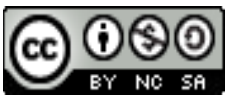

Copyright: (c) 2020 Hernández-Viveros, A.S.; Guarneros-Reyes E. \& Espinoza-Zepeda, A.J.

Este es un artículo de acceso abierto distribuido bajo los términos de la licencia Creative Commons Reconocimiento-NoComercial 4.0 Internacional, por lo que su contenido gráfico y escrito se puede compartir, copiar y redistribuir total o parcialmente sin necesidad de permiso expreso de sus autores con la única condición de que no se puede usar con fines directamente comerciales y los términos legales de cualquier trabajo derivado deben ser los mismos que se expresan en la presente declaración. La única condición es que se cite la fuente con referencia a la Revista Digital Internacional de Psicología y Ciencia Social y a sus autores 


\section{TABLA DE CONTENIDO}

INTRODUCCIÓN

Simulación digital, 483

Simulador Moodle como herramienta de apoyo al plan de estudios, 484

NATURALEZA DE LA INNOVACIÓN

484

Simulador digital en Moodle para la enseñanza del vocabulario infantil dirigido a alumnos del SUAYED, 484

\section{CARACTERÍSTICAS DE LA INNOVACIÓN}

Simulación por ramificación, 485

Procedimiento del desarrollo del diseño del simulador, 487

Evaluación, 488

Administración, 488

RANGO DE APLICACIÓN DE LA INNOVACIÓN

Competencias transversales, 489

\section{FUNCIONAMIENTO DE LA INNOVACIÓN}




\section{INTRODUCCIÓN}

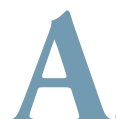

I ser estudiantes de Psicología, ya sea en modalidad presencial o a distancia, se hacen prácticas que fomenten sobre dichas competencias profesionales que, de acuerdo con el plan de estudios, deberán ser adquiridas mediante un entrenamiento profesional en escenarios reales (Silva, Peñalosa, Aragón y Contreras, 2005). Dichas prácticas regularmente se desarrollan por medio de una solicitud que el estudiante debe presentar a alguna institución educativa, en la cual le permitan observar el trabajo del profesional de la psicología infantil, y en algunos casos participar con alguna actividad que antes haya planificado.

Es necesario mencionar que las prácticas que desarrollan los estudiantes de Psicología es, para muchos de ellos, el primer encuentro con un escenario real, y por ello no se tienen los conocimientos y experiencias necesarias para lograr un desempeño exitoso durante las prácticas; asimismo se corre el riesgo de mala toma de decisiones en el campo experimental, o un mal manejo de las emociones, pues el estudiante aún no conoce las diversas situaciones a las que pueda enfrentarse.

En esta misma línea, buscando soluciones en las tecnologías para la experiencia enseñanza-aprendizaje podrían usarse, aunque no es común, los recursos virtuales, de realidad virtual, 3D, realidad aumentada, de simulación virtual, y nuevas tecnologías que puedan aplicarse a la docencia e investigación, y que a su vez puedan estar libremente al alcance de la comunidad estudiantil como apoyo a su experiencia formativa. Por ejemplo, Maneru (2012) indica que la simulación en un ambiente educativo es un entrenamiento que ofrece al estudiante la oportunidad de efectuar una práctica constante para lograr la adquisición de destrezas psicomotrices familiarizándose con instrumentos y equipos tecnológicos, por medio de la cual adquiere experiencia en el reconocimiento de problemas; sin embargo estos escenarios para el aprendizaje en Psicología Sistema de Universidad Abierta y Educación a Distancia SUAyED no son comunes; los materiales más usuales son las antologías que se constituyen de documentos en PDF, que son capítulos de libros, o artículos que los tutores seleccionan para subirlos a la plataforma; las actividades más comunes que incluso se encuentran en el plan de estudios son la síntesis, la glosa, el ensayo y la participación.

Por ejemplo una de las prácticas que realizan los estudiantes de psicología en el ámbito infantil es sobre vocabulario en niños. Un estudio de Morales (2013) destaca la importancia del entrenamiento del vocabulario infantil en el aula, pues aseguran que son necesarias evaluaciones precisas y de programas para mejorar el desarrollo del vocabulario de los niñas y niños mediante programas de intervención sistematizados, ya que a pesar de que el profesorado es consciente de la importancia de la adquisición del vocabulario, a veces no le dedica suficiente atención a este aspecto porque se trata el componente léxico sin mucha sistematicidad y sin una tipología amplia de actividades claramente encaminadas a la consecución de dicho objetivo; por ello plantean necesario planificar intervenciones que presenten mejor contextualización y una tipología más amplia de actividades que permita la asimilación de las nuevas unidades léxicas. Se entiende entonces entonces que la manera en que evoluciona el vocabulario infantil está asociado a la manera en que éste se entrena, por lo que es indispensable que el psicólogo en formación tenga presente que esta es un área en la que deberá desarrollar competencias a lo largo de su carrera.

\section{Simulación digital}

Aquí es conveniente destacar la utilidad de las tecnologías digitales, y sobre todo de la simulación digital, como herramienta de apoyo para el estudiante de Psicología de sistemas presenciales o a distancia, quienes como se ha comentado, al hacer sus prácticas en escenarios reales es probable que enfrente situaciones hasta ese momento desconocidas y nuevas; también es necesario utilizar herramientas virtuales que puedan servir como auxiliar preventivo de errores durante dichas prácticas.

Dentro de estos ambientes virtuales están los simuladores, los cuales se definen como una poderosa herramienta que permite el análisis, estudio, evaluación de situaciones y toma de decisiones que de otra manera serían difíciles de analizar, los cuales están diseñados para la resolución de problemas por medio de modelos de un sistema hecho para que el usuario pueda comprender el comportamiento de éste, y evaluar las distintas estrategias operativas del sistema en estudio (Cataldi, Lage y Dominighini, 2013).

Una de las grandes ventajas de la simulación en ambientes virtuales es que el alumno puede verse inmerso en un ambiente de práctica, mismo al que muchas veces no tiene fácil acceso en la realidad, o como en este caso, a modo de preparación antes de presentarse en un ambiente real de práctica.

Solbes (2016) hace una revisión de la realidad virtual aplicada a trastornos infantiles, tales como fobia a volar, acrofobia, fobia social, fobia a las arañas, claustrofobia, trastornos alimentarios y estrés postraumático, 
mencionando que su uso cada vez va en aumento debido al mayor acceso a las TIC disponibles actualmente.

Entonces se puede ver que los ambientes virtuales han contribuido al tratamiento de algunos desórdenes psicológicos; sin embargo, podemos asegurar que así como dichos ambientes virtuales son utilizados en el área de psicología clínica, también deben ser utilizados desde el proceso de la formación profesional del psicólogo, otorgándole la oportunidad al estudiante de acercarse a estas nuevas herramientas virtuales y de utilizarlas para su beneficio durante el proceso de aprendizaje.

Al utilizar simuladores que recreen una realidad en un ambiente controlado se permite que el estudiante obtenga mayor seguridad en sus conocimientos, emociones y de experimentar, incluso en repetidas ocasiones y en distintas circunstancias, hasta que esté preparado para enfrentar un ambiente real; le permite también tener realimentación de manera instantánea y reforzar sus habilidades de toma de decisiones sin que tenga ese miedo o estrés de poder equivocarse o dañar a algún tercero por un mal desempeño.

\section{Simulador Moodle como herramienta de apoyo al plan de estudios}

El prototipo se diseñó en la plataforma de Moodle (Guarneros, 2017), pues éste es un recurso flexible, económico y libre que se puede utilizar por una plantilla grande de estudiantes, lo cual es ideal para cubrir las necesidades y dar soporte a los estudiantes de Psicología en sus modalidades abierta, a distancia y escolarizada. La plataforma educativa Moodle LabVE Laboratorio Virtual de Enseñanza (http://desarrolloinfantil.uno/labovirtual) es un Learning Management System (LMS), esto es un sistema de gestión del aprendizaje que permite controlar tanto los contenidos como los usuarios que interactúan dentro de él. Moodle tiene herramientas de comunicación y seguimiento de actividades de los usuarios, pues uno de sus principales beneficios es proporcionar a los educadores mejores herramientas para administrar y promover el aprendizaje (Marín y Maldonado, 2011).

Entre las aportaciones de Moodle al proceso enseñanza-aprendizaje, destacan: 1) funcionalidades didácticas sofisticadas y ricas en opciones; 2) el diseño modular y la facilidad en el diseño de la interfaz del usuario permite que sea más utilizado que el de sus competidores, y 3) el grado de apertura y el dinamismo del proyecto es muy elevado, pues es manejado por una comunidad abierta y amplia de usuarios dispuestos a colaborar con el mejoramiento del entorno por medio de módulos y características adicionales en un periodo breve (Marín y Maldonado, 2011).
Por lo anterior, el simulador que se diseñó en este trabajo contiene estas propiedades de Moodle que Marín y Maldonado (2011) indican y se utiliza como una herramienta extra al alcance de cualquier alumno de la carrera de Psicología en sus modalidades abierta, a distancia o escolarizada; y por la flexibilidad y el soporte que tiene la plataforma de Moodle, ésta puede ser usada no solo por la Facultad donde se creó el simulador, sino que puede compartirse con estudiantes de cualquier parte del mundo.

\section{Naturaleza de la inNovación}

Es necesario retomar el problema planteado en el proyecto PAPIME PE307717, en el cual se enmarca este trabajo y del que se recibió el financiamiento de beca por parte del Programa de Apoyo a Proyectos para la Innovación y Mejoramiento de la Enseñanza (PAPIME). ¿Cómo acercar a los estudiantes inexpertos en situaciones reales a la investigación o el ejercicio profesional y que se les permita ser más competentes en el ámbito de incidencia de la Psicología? (Guarneros, 2017). En este caso, para apreciar cómo se expande y promueve el desarrollo del vocabulario infantil para que los estudiantes de Psicología hagan propuestas de investigación o de intervención en que se pueden utilizar simuladores de los procesos evolutivos que la literatura científica reporta (Guarneros, 2017); por su parte, Cataldi, Lage y Dominighini (2013) mencionan que hay algunas situaciones difíciles de recrear para su estudio, y por ello el uso de los simuladores digitales es primordial, pues estos programas son capaces de reproducir situaciones y fenómenos reales.

\section{Simulador digital en Moodle para la enseñanza del vocabulario infantil dirigido a alumnos del SUAyED}

Con la ayuda de simuladores Moodle se pretende que el estudiante tenga la posibilidad de aprender a partir del error o a partir de modificar los valores de las variables para inferir en el comportamiento del modelo, porque le es de utilidad para ensayar o experimentar diferentes hipótesis, permitiéndole verse más involucrado en su proceso de aprendizaje, pues tiene el poder de decisión al manejar el simulador, y con esto observar sus resultados y actuar en consecuencia.

El desarrollo que se ha hecho permite cumplir los objetivos 3, 4 y 5 del proyecto PAPIME PE307717, los cuales consisten en los siguientes aspectos.

- Analizar las características de software de simulación y diseñar la interfaz en Moodle para los simuladores.

- Diseñar los simuladores en Moodle con una estructura de presentación, navegación, verificación, ma- 
nipulación y evaluación integradas en una interfaz gráfica integral según el prototipo.

- Evaluar, por medio de expertos, las cualidades de los simuladores en Moodle para modelar la evolución del lenguaje infantil y si cumple sus objetivos.

Articulate (2014) indica que el ser humano por naturaleza es curioso y le gusta explorar nuevos terrenos, por lo que en un ambiente enseñanza-aprendizaje el uso de recursos multimedia, como videos y fotografía, pueden ser muy útiles para asimilar la información; este tipo de interacciones facilitan la transmisión del mensaje mientras se mantiene interesado al usuario. Si no se cuenta con estos recursos multimedia, éstos pueden ser creados con un ordenador y desplazarse por medio de diferentes rutas con sólo pulsar una tecla. El mismo autor asegura que una manera de enganchar al usuario es mediante el uso de preguntas, pues éstas logran impactar en lo que él verá después, o les ayuda a practicar nuevas habilidades incluso con sólo preguntar “¿Qué harías en esta situación?".

\section{Características de la innovación}

Es evidente que con el uso correcto de las configuraciones se puede crear interactividad; una manera en que estas interacciones se tornan significativas es por la relevancia que este aprendizaje trae para el usuario, presentándoles situaciones con las cuales tiene que enfrentarse en la vida real, pues sólo necesita pensar en lo que ya ha aprendido y tomar decisiones a partir de sus nuevos conocimientos. El paso siguiente es recrear un escenario y situaciones lo más parecidas a lo real; se crean situaciones de interactividad por medio de preguntas basadas en situaciones reales que obligan al usuario a pensar en una buena decisión; por lo regular no hay opciones del todo correctas sino sólo diferentes consecuencias, pues cada decisión creará un nuevo escenario (Articulate, 2014).

\section{Simulación por ramificación}

Se entiende que la simulación por ramificación conlleva una libre elección para el usuario de acuerdo con las opciones que se le proporcionan, y cada una de dicha decisiones le llevan a una nueva situación derivada como consecuencia; a su vez, después de esta consecuencia se le presentan nuevas opciones, con nuevas consecuencias cada una, abriéndose diferentes rutas, las cuales terminan siempre en una última situación que es el término de la actividad o situación propuesta, pero con diversas posibilidades de Ilegada. Dentro de este simulador digital en Moodle para la enseñanza del desarrollo del vocabulario infantil se le proporcionan dos opciones de respuesta al usuario, de las cuales debe ser capaz de decidir entre una de ellas y en seguida recibir realimentación de la consecuencia que derivó de su decisión; si ésta no fue la más eficaz, el usuario tiene la opción de revalorar su decisión y seleccionar otra ruta que de nuevo lo encamine a una ruta más acertada; cada una de las consecuencias abre dos nuevas posibilidades de elección, y cada decisión que se toma tiene su propia consecuencia, creando una ruta que, cualquiera que sea, siempre tiene el mismo final.

Round, Conradi y Poulton (2007) consideran que la simulación por ramificación es un método donde, para el diseño, es necesario un esfuerzo para crear un largo pero limitado número de opciones, permitiendo al usuario elegir dos o tres malas decisiones antes de percibir su error; después de tomar una mala decisión, se le da la opción de tomar la decisión correcta siempre y cuando la mala decisión no represente un peligro. Estos mismos autores desarrollaron este método para crear un paciente virtual, y para ello explican que los pasos para la simulación por ramificación deben ser los siguientes.

- Elegir el tipo de paciente. Aquí debe elegirse el escenario que necesita evaluación e investigación que sirva para dirigir manejo de los pasos.

- Especificar los nodos clave. Son etapas que funcionan como una puerta de entrada a la siguiente etapa del caso.

- Crear una ruta ideal. Ésta no tiene que ser la única, dentro del caso, pero sí dará la pauta para saber cuántos pasos deben programarse; la limitación de nodos es de nuevo un dispositivo para restringir el número potencial de situaciones entre cada nodo, porque para cada elección correcta habrá otras opciones, cada una de las cuales conducirá a otras opciones (figura 1).

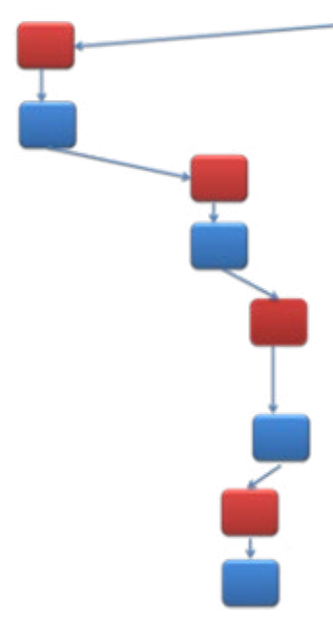

Figura 1.

Imagen representativa de la interconexión de nodos en la ruta ideal. 
- Colocar nodos interconectados y ramificados. Éstos representan situaciones por las que se puede pasar (figura 2).

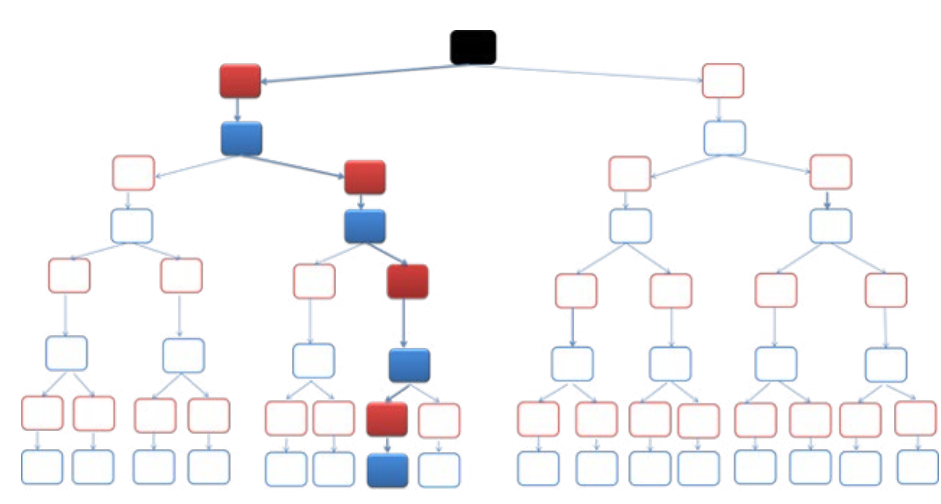

Figura 2.

Representación de un mapa completo en ramificación.

- Decidir qué representa cada caja y cuáles decisiones las conectan. Es necesario pensar en lo que puede ser más razonable en una situación real y utilizarlo como base para nombrar las decisiones.

- Reducción del caso. Las ramificaciones que no tienen una salida necesitan una explicación y ser redireccionadas a otro nodo anterior o al inicio.

- Nombrar cada caja. Es necesario un sistema de nombres lógicos y cortos.

- Elaborar una hoja de cálculo. De esta manera el diseñador del simulador puede comunicarse con un técnico para describir el texto de cada situación a la que será dirigido el usuario después de cada decisión.

- Insertar una interfaz de usuario. Se puede hacer sólo elaborando páginas HTLM individuales, diseñando un esquema XML utilizando un reproductor adecuado.

- Enriquecer con medios. Una vez que el esqueleto de la situación que se va a simular está concluido, se pueden utilizar otras herramientas como fotografía, video, sonido, vincular otras fuentes de información (como materiales y cursos en línea), todo dependiendo del tiempo y recursos con que se cuente (Round, Conradi y Poulton, 2007). Para el diseño y configuración del simulador digital en Moodle para la enseñanza del desarrollo del vocabulario infantil se siguieron los mismos pasos de la simulación por ramificación antes mencionados, modificándolos con un enfoque dirigido a un ambiente escolar, escogiendo, en lugar de un tipo de paciente, un escenario educativo, siguiendo los pasos que a continuación se detallan.

Durante el desarrollo de este simulador digital en Moodle se logró el análisis, capacitación y ejecución de los diferentes softwares, aplicaciones y plataformas que se utilizaron para la simulación, tales como Moodle, Go Animate y Grabador de Voz; también se hizo el diseño de la interfaz en Moodle para el simulador primero mediante la capacitación en el uso de ésta y después con su aplicación para establecer el montaje del simulador. El diseño del simulador en Moodle se hizo cuidando que la estructura y presentación fueran amigables a la vista, de fácil manipulación y navegación para el usuario, todo en una interfaz gráfica integral de acuerdo con el prototipo.

Por último la evaluación se hizo en tres fases, la primera con un intercambio y revisión entre colaboradores del proyecto, lo que sirvió como primer filtro para eliminar errores y escuchar algunos comentarios y sugerencias de los integrantes del equipo, mismos que una vez resueltos dieron lugar a la segunda fase, la cual fue un filtro que consistió en otra revisión de expertos en el tema, quienes identificaron diferentes detalles y aportaron sugerencias con base en sus conocimientos de diseño de cursos en esta plataforma de Moodle; para finalizar, el producto del simulador quedó sujeto a revisión por los administradores del Laboratorio Virtual.

El simulador digital en Moodle se desarrolló en esta plataforma debido a las grandes ventajas que tiene, como que es una plataforma mundialmente aprobada, su diseño capaz de soportar tanto la enseñanza como el aprendizaje, debido a un extenso conjunto de herramientas enfocafas en el estudiante y ambientes de aprendizaje colaborativo; es una plataforma fácil de usar, pues tiene características de arrastrar y soltar; es gratuita y no tiene cargos de licencia, cualquier persona puede descargarlo y hacer modificaciones, extensiones y adaptaciones porque su código abierto siempre está actualizado, pues su programa de código abierto significa que Moodle es continuamente revisado y mejorado para adecuarse a las necesidades actuales y cambiantes de sus usuarios; se encuentra en una gran variedad de idiomas; al momento tiene traducción a más de 120 idiomas; es flexible, seguro y privado porque ha implementado procesos del desarrollo de Moodle y software para protección contra acceso no autorizado, pérdida de datos y mal uso; puede utilizarse en cualquier momento y en cualquier dispositivo electrónico, con una interfaz por defecto compatible con dispositivos móviles y compatibilidad cruzada con diferentes navegadores de internet; el contenido en la plataforma Moodle es fácilmente accesible y consistente a lo ancho de diferentes navegadores y dispositivos. La plataforma Moodle donde se encuentra el simulador es el Laboratorio Virtual de Enseñanza. 


\section{Procedimiento del desarrollo del diseño del simulador}

El primer paso fue la elaboración de un prototipo que pudiera servir como modelo de aplicación, como herramienta de práctica. La metodología que se usó fue el diseño de ambientes virtuales de aprendizaje basada en On Line Learning \& Training o Aprendizaje y Entrenamiento en Línea (Mendoza y Galvis, 1999), el cual permitió construir, modelar y documentar todas las partes que contenía el Simulador Digital en Moodle para la Enseñanza del Desarrollo del Vocabulario Infantil.

Con base en el éxito de las etapas de diseño utilizadas en proyectos anteriores por parte del Laboratorio Virtual de Enseñanza, y a los requerimientos específicos de los simuladores en Moodle, estas fases se conformaron de la siguiente manera.

Capacitación. Etapa que dio arranque al diseño del simulador en Moodle, pues el equipo de colaboradores trabajó por primera vez con este tipo de softwares, iniciando con una capacitación general de cada uno de éstos y que se utilizaron durante el diseño de dicho simulador.

Análisis. En éste participaron tanto profesores como estudiantes y colaboradores del proyecto; éste se desarrolló mediante la revisión minuciosa de las diferentes necesidades, especificando las características instruccionales (como el propósito y objetivos de los simuladores en Moodle, contenidos viables y medios que lo apoyan, y quiénes y cómo son los recursos tecnológicos de las personas involucradas en el sistema); los hallazgos de la literatura con que se tuvieron que definir los modelos evolutivos encontrados, recapitular los hallazgos a modo de estado del arte y diagramas que debían seguir los simuladores en Moodle especificados desde el punto de vista de los estudiantes; dichos diagramas se definen como una notación gráfica, y debían describir tanto la especificación completa y suplementaria de los requerimientos de los simuladores - lo que hace el estudiante- como lo que hace el simulador por medio de las diferentes rutas mientras interactúan.

Diseño. Durante esta fase el diseño siguió las especificaciones del análisis y se esquematizaron en 1) el diseño de los simuladores en Moodle; 2) diseño de interfaz y funcionalidad en Moodle; 3) sistema de navegación; 4) retroalimentaciones; 5) estructura de navegación y de presentación, y 6) diseño de interfaz gráfica (botones, pestañas, títulos, definir hojas de estilo, tipo de imágenes, etcétera).

Desarrollo. En esta fase se elaboró y construyó el prototipo. Las simulaciones Moodle son formas que ofrece internet para modelar el mundo y concretar los modelos abstractos de las teorías e investigaciones, por lo que en esta etapa se configuró el ambiente con las ricas herramientas que ofrece Moodle: su portabilidad, compatibilidad, fácil actualización y robustos sistemas de evaluación, integrando los elementos del diseño HTML, multimedia y digitales que el mismo Moodle.

Para acceder al simulador, el usuario debe tener una contraseña proporcionada por el Laboratorio Virtual de Enseñanza; una vez que se tiene acceso, el usuario podrá diseñar un avatar, el cual podrá ser femenino o masculino, dependiendo el personaje que quiera representar en el simulador en primera persona; posterior a esto, y una vez que comience con el desplazamiento por el simulador, en la parte principal se tendrá un video explicativo de los objetivos y utilidad de dicho simuador; el video, al igual que los demás videos utilizados en el simulador, fue tomado del repertorio de videos hechos con ayuda de la aplicación Go Animate y fue incrustado de modo directo en Moodle desde dicha aplicación en código HTML, esto para que cada uno de los videos abran directamente desde el simulador y no desde la página de Go Animate (figura 3).

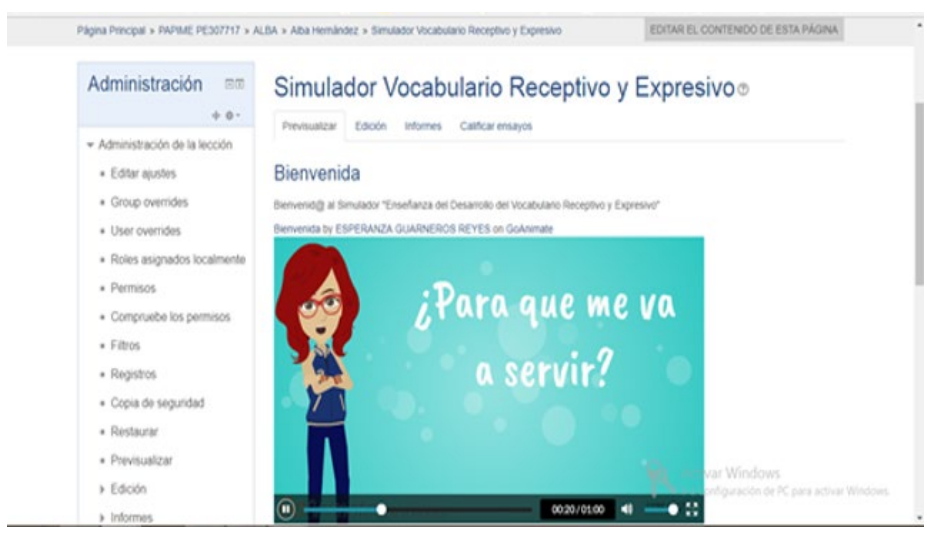

Figura 3.

Primera página de contenido de bienvenida del Simulador.

Después se inicia con el planteamiento de la situación simulada, que sirve de preámbulo para dar un panorama general del comienzo de la toma de decisiones que el usuario tiene que efectuar, las cuales se presentan en forma de video cuestionando al usuario (" ¿Ahora que harás?"), y ofreciendo sólo dos opciones de respuestas, planteadas por medio de un video por cada decisión; una vez que ha decidido, comienza el enrutamiento de su toma de decisiones, mostrando - después de cada elección - una consecuencia acorde a ésta, por medio de un video; aunado a la consecuencia, el usuario podrá ver en el video una retroalimentación inmediata correspondiente a su decisión, la cual, en caso de ser la más eficaz, le exhorta a continuar y seguir desplazándose por el simulador; por lo contrario, si es la menos acerta- 
da, le sugiere tomar otra decisión, o lo regresa a alguna previa toma de decisión para que pueda revalorar de nuevo su elección. La configuración de las decisiones y sus opciones se desarrollaron por medio de preguntas de opción múltiple localizadas en el banco de preguntas y después trasladadas a la lección del simulador, en las que se agregaron los videos explicativos de cada una de las dos opciones, y mediante la configuración de los botones de salto, cada una de las opciones redirecciona a una página de contenido, que en este caso es la consecuencia de dicha decisión; en esta parte la nomenclatura correcta de las decisiones y de las consecuencias fue muy importante para la configuración (figura 4).

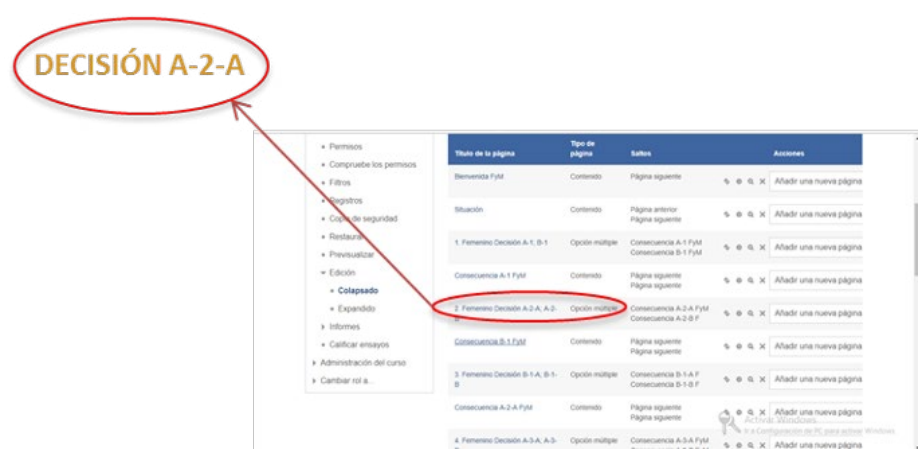

Figura 4.

Configuración de enrutamiento de la toma de decisiones.

De esta manera el usuario interactúa con las situaciones, decisiones y consecuencias por medio del simulador hasta llegar a un final que en todos los casos será el mismo; pues todas las rutas llegan al mismo fin; sin embargo se espera que, con base en su preparación, el estudiante sea capaz de identificar la ruta más eficaz, ya que puede seguir una trayectoria corta o más larga, dependiendo de las decisiones que va tomando en el recorrido.

\section{Evaluación}

La valoración en este tipo de metodología se planteó cíclica; en cada etapa se hicieron ajustes; con la evaluación se determinaron las fallas a nivel de análisis, diseño y desarrollo. Participaron todos los integrantes. Se formularon instrumentos de evaluación, los cuales consistían en captura de pantalla referente a la corrección solicitada, un breve comentario de la modificación sugerida y el link de la página de contenido en que se encontró el error o sugerencia. Se planearon las siguientes evaluaciones de cuatro dimensiones.

- Evaluación del experto del área. Investigadores del lenguaje infantil y ambientes virtuales hicieron el pilotaje y entregaron comentarios en el formato indicado.
- Evaluación de prototipo rápido. Profesionales en desarrollar ambientes digitales de aprendizaje pilotearon el simulador y entregaron comentarios y sugerencias en el mismo formato.

- Prueba de amigabilidad, funcionalidad y navegabilidad con alumnos.

- Aprendizajes de los alumnos.

Este trabajo cubrió su reporte hasta la evaluación del experto del área (investigadores del lenguaje infantil y evaluación de prototipo rápido, quienes fueron profesionales en desarrollar ambientes digitales de aprendizaje); basados en sus comentarios se hicieron las modificaciones correspondientes y se dejó el simulador digital en manos de los integrantes del Laboratorio Digital de Desarrollo Infantil.

\section{Administración}

En esta fase se hizo la revisión del funcionamiento y gestión de los procesos del manejo de los simuladores en Moodle, como registros de rendimiento, sistemas de almacenamiento, logs, administrar las trayectorias de aprendizaje y la actividad de los usuarios.

\section{RANGO DE APLICACIÓN DE LA INNOVACIÓN}

Los ambientes digitales y de simulación son muy importantes porque, como hemos revisado a lo largo de este trabajo, los recursos tecnológicos avanzan de modo muy rápido y se renuevan de manera constante; la educación no tiene por qué ser la excepción, y tiene que estar a la altura; por ello consideramos que el Simulador en Moodle Para la Enseñanza del Desarrollo del Vocabulario Infantil es un gran auxiliar como apoyo a la docencia en el campo de la Psicología. Por ejemplo, Contreras y Carreño (2012) indican que los simuladores digitales tienen las siguientes utilidades.

- Ofrecer habilidades al estudiante en el proceso enseñanza-aprendizaje que sean compatibles con el entorno de trabajo y en sus prácticas de investigación.

- Ayudar al docente moderno a crear comunicación maestro-máquina estudiante por medio de interfaces visualizadas de manera gráfica por el cerebro.

- Proporcionar al estudiante medios de autoaprendizaje y automotivación.

- Crear comunicación maestro-estudiante y estudiante-estudiante por medio de la simulación interactiva, que puede ser complementada con la retroalimentación para enriquecer el proceso educativo.

- Flexibilidad en los horarios de trabajo. Los autores también sostienen que el docente puede ser capaz de crear entornos de aprendizaje virtual utilizando la 
configuración de las variables de interacción maestro, estudiante, tecnología educativa y medio ambiente.

\section{Competencias transversales}

Con el uso de la simulación, en el usuario se promueven las competencias transversales tales como el conocimiento científico teórico y práctico; habilidades y destrezas para actuar de modo adecuado en la práctica, la capacidad para resolver problemas de manera contrastada y científica, y la práctica para transferir su conocimiento a nuevas situaciones; se promueve también la capacidad para argumentar y justificar de modo científico su acción, el pensamiento crítico para actuar —articulando teoría y práctica-, la búsqueda del cambio de la práctica y de los contextos como oportunidad para la transformación de los mismos, la capacidad para trabajar desde la incertidumbre, la capacidad para buscar opciones ante las nuevas situaciones que se les plantean, la capacidad para evaluar la acción profesional en todas sus dimensiones (humana, social, económica, efectividad, etcétera), y la capacidad para valorar las consecuencias éticas de sus acciones; también se promueve la capacidad para trabajar en equipo, así como la capacidad para comprometerse con su entorno profesional, la capacidad para trabajar en contextos interculturales, para comunicarse con el otro, para compartir conocimientos y recursos, y para cooperar con los otros de manera interdisciplinar para construir conocimiento de modo colectivo preservando la identidad de cada uno (Méndez, 2014). En el caso del simulador diseñado se buscó dar atención a estas demandas para acercar al alumno de Psicología del SUAyED a un ambiente educativo de práctica por medio de dichas competencias.

\section{Funcionamiento de La inNovación}

El uso de simuladores sistemáticos durante el proceso de formación técnica y profesional está relacionado con la mejoría de la comprensión conceptual, facilitando el aprendizaje y preparando a los futuros profesionistas con mejores habilidades y competencias, mismas que se reflejan en un perfil profesional más completo y de calidad (Díaz-Forero, 2012). Por ello es necesario reiterar la necesidad de incluir al psicólogo en formación del SUAyED en estos ambientes virtuales, para que explote al máximo los beneficios que éstos ofrecen y que le ayuden a tener una formación más completa, vanguardista y acorde con los tiempos inmersos en avances tecnológicos que le ha tocado vivir. Beneficiar al estudiante de Psicología del SUAyED con un simulador digital que promueva las competencias transversales y sus funciones cognitivas, que ya hemos explicado, puede dotarlo de seguridad y herramientas básicas para el ejercicio de sus prácticas de campo. Entonces podemos sostener que, después de la revisión con los expertos en el tema de diseños de cursos virtuales, y de los ajustes que hicieron los administradores del Laboratorio Virtual de Enseñanza, el uso del Simulador en Moodle para la Enseñanza del Desarrollo del Vocabulario Infantil da respuesta a los requerimientos formativos del universitario del SUAyED, pues este simulador es dinámico, sencillo de utilizar, intuitivo y sobre todo está basado en casos reales que proporcionan mayor sensación de realismo e inmersión; también contribuye al proceso de aprendizaje del estudiante, pues para que éste sea un egresado competitivo debe saber desenvolverse en un ambiente innovador, en donde el uso de las nuevas tecnologías es cada vez más frecuente.

\section{CONCLUSIONES}

Como hemos revisado, los alcances de la simulación y del uso de las tecnologías de información y comunicación (TIC) no deben parecernos obvios; al contrario, este es un terreno que debe ser explorado desde una perspectiva educativa que sirva como una herramienta constante y al alcance de los estudiantes, quienes deben ser más competitivos y saber valerse de todas las herramientas tecnológicas de libre acceso con que se cuenta en estos tiempos; el estudiante universitario de hoy está acostumbrado a tener información de modo rápido y confiable mediante las TIC; sin embargo, es necesario proporcionarle nuevas posibilidades que puedan enriquecer $y$ fortalecer sus conocimientos para ser aplicados en su proceso de aprendizaje. La tecnología hoy avanza a pasos agigantados; basta tomar el ejemplo de los teléfonos celulares que, con el paso del tiempo, ya no son utilizados sólo para hacer Ilamadas telefónicas, sino que han ido evolucionando al ir integrando funciones cada vez más novedosas y complejas, hasta llegar a ser los dispositivos inteligentes que son ahora. Es por ello que al hablar de tecnología se debe ser consciente de que ésta no es estática y que es necesario que la exploremos y explotemos al máximo, para sacarles el mayor provecho, sobre todo en el ámbito que nos concierne, que es el formativo de los futuros profesionistas. Para finalizar se puede decir que el Simulador Digital en Moodle para la Enseñanza del Desarrollo del Vocabulario Infantil diseñado para entrenar al estudiante de Psicología está muy apegado a las situaciones que éste encontrará en una práctica real, por lo que puede sentirse familiarizado con el planteamiento que se le hace y verse obligado a eliminar de manera lúdica las carencias de experien- 
cia y a desarrollar las competencias necesarias para su formación. Por ello esta propuesta es elaborada con la mejor intención de ser un elemento de ayuda que pueda cubrir dichas necesidades, sobre todo considerando el aprendizaje de un aspecto necesario que estudian los alumnos de Psicología en el ámbito educativo (el desarrollo del vocabulario), el cual fue ejemplo en este trabajo respecto a cómo plantear situaciones que debe evaluar el estudiante cuando atiende niños que están desarrollando su vocabulario, con qué actividades puede fomentar el desarrollo, o como detectar algún problema, así como atender situaciones inesperadas.

En conclusión, se logró analizar las características de software de simulación y diseñar la interfaz en Moodle para los simuladores, así como un diseño exitoso del simulador en Moodle con una estructura de presentación, navegación, verificación, manipulación y evaluación integradas en una interfaz gráfica según el prototipo; por último, se logró evaluar, junto con expertos, las cualidades de los simuladores en Moodle para modelar la evolución del lenguaje infantil cumpliendo los objetivos de herramienta de práctica para estudiantes de Psicología, ya sea en su modalidad presencial o a distancia.

\section{Referencias}

Articulate (2014). 5 Highly Effective Strategies for Creating Engaging E-Learning. Recuperado de: https://community. articulate.com/e-books/5-highly-effectivestrategies-forcreating-engaging-e-learning

Cataldi, Z., Lage, F. J., \& Dominighini, C. (2013). Fundamentos para el uso de simulaciones en la enseñanza. Revista de Informática Educativa y Medios Audiovisuales, 10(17), 8-16. Recuperado de: http://laboratorios.fi.uba.ar/lie/ Revista/Articulos/101017/A2mar2013.pdf

Contreras, G. A., \& Carreño, P. (2012). Simuladores en un ambiente educativo: Un recurso didáctico para la enseñanza. Ingenium Revista de la Facultad de Ingeniería, pp. 107-119. Recuperado de: https://dialnet.unirioja.es/ servlet/articulo?codigo $=5038479$

Díaz-Forero, J. (2012). Simulación en entornos virtuales. Una estrategia para alcanzar "aprendizaje total", en la formación técnica y profesional. Revista Latinoamericana de Estudios Educativos, XLII (2), 49-94. Recuperado de: $\quad$ http://www.cee.edu.mx/revista/r2011-2020/r texto/t_2012_2_03.pdf
Guarneros, E. (2017). Diseño de simuladores en Moodle para la enseñanza de la manera en que evoluciona el lenguaje infantil. PE307717. México: DGAPAUNAM.

Maneru, G. (2012). La adquisición de habilidades y competencias en el Centro de Simulación Médica. De la teoría a la práctica. Revista Internacional de Humanidades, 1(2), 195-217. Recuperado de: https://www.unav.edu/ documents/29044/2996238/adquisicion-habilidadescompetencias-centro-simulacion.pdf

Marín, V., \& Maldonado, G. A. (2011). El alumnado universitario coldobés y la plataforma virtual Moodle. Pixel-Bit. Revista de Medios y Educación, pp. 121-128. Recuperado de: http://www.redalyc.org/articulo.oa?id=36816200009

Méndez, M. (2014). Competencias transversales: Una herramienta fundamental para un excelente desempeño en el puesto de trabajo. Universidad Militar, Nueva Granada. Recuperado de: http://repository.unimilitar. edu.co/bitstream/10654/10794/1/MendezAlvaradoM arisol2014.pdf

Mendoza, P., \& Galvis, A. (1999). Ambientes virtuales de aprendizaje: Una metodología para su creación. Informática Educativa, 12(2), 295-317. Recuperado de: $\quad$ https://staff.concord.org/ agalvis/AG site/Assets/ publications/Articulos/1999\%20AVA-METODOLOGIA\%20 RIE\%2012(2).pdf

Morales, F. (2013). Eficacia de un programa de entrenamiento en el vocabulario en niños. Revista de Investigación en Logopedia 3. Recuperado de: https://cvc.cervantes.es/ ensenanza/biblioteca ele/publicaciones centros/PDF/ rio_2007/49_siqueira.pdf

Round, J., Conradi, E., \& Poulton, T. (2007). A Workable Model for Virtual Patient Design. In Proceedings of the SOLSTICE 2007 Conference, Researching eLearning for Innovation and Development. Recuperado de: http://www.jround. co.uk/virtual_patients/papers/workable_vp.htm

Silva, A., Peñalosa, E., Aragón L., \& Contreras, O. (2005). Plan de estudios vigente de la Licenciatura en Psicología del Sistema de Universidad Abierta y Educación a Distancia. FES-Iztacala, UNAM. Recuperado de: https://suayed. iztacala.unam.mx/wp-content/uploads/2015/02/Plan-deEstudios-Lic-Psicologia-a-Distancia.pdf

Solbes, A. (2016). Revisión bibliográfica sobre la realidad virtual aplicada a los trastornos infantiles. Universitat Jaume I. Recuperado de: http://repositori.uji.es/xmlui/ handle/10234/162176 
Innovación y desarrollo tecnológico | Simulación digital en Moodle ... Hernández-Viveros, Guarneros-Reyes ę Espinoza-Zepeda

Meta-Análisis

del Artículo

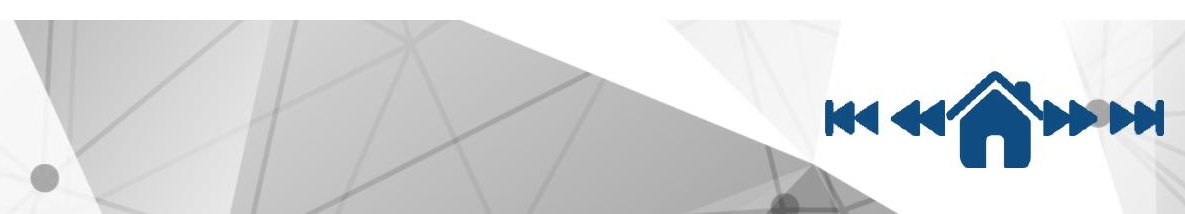




\section{Dimensión Cuantitativa}

\section{Perfil de Evaluación entre pares}
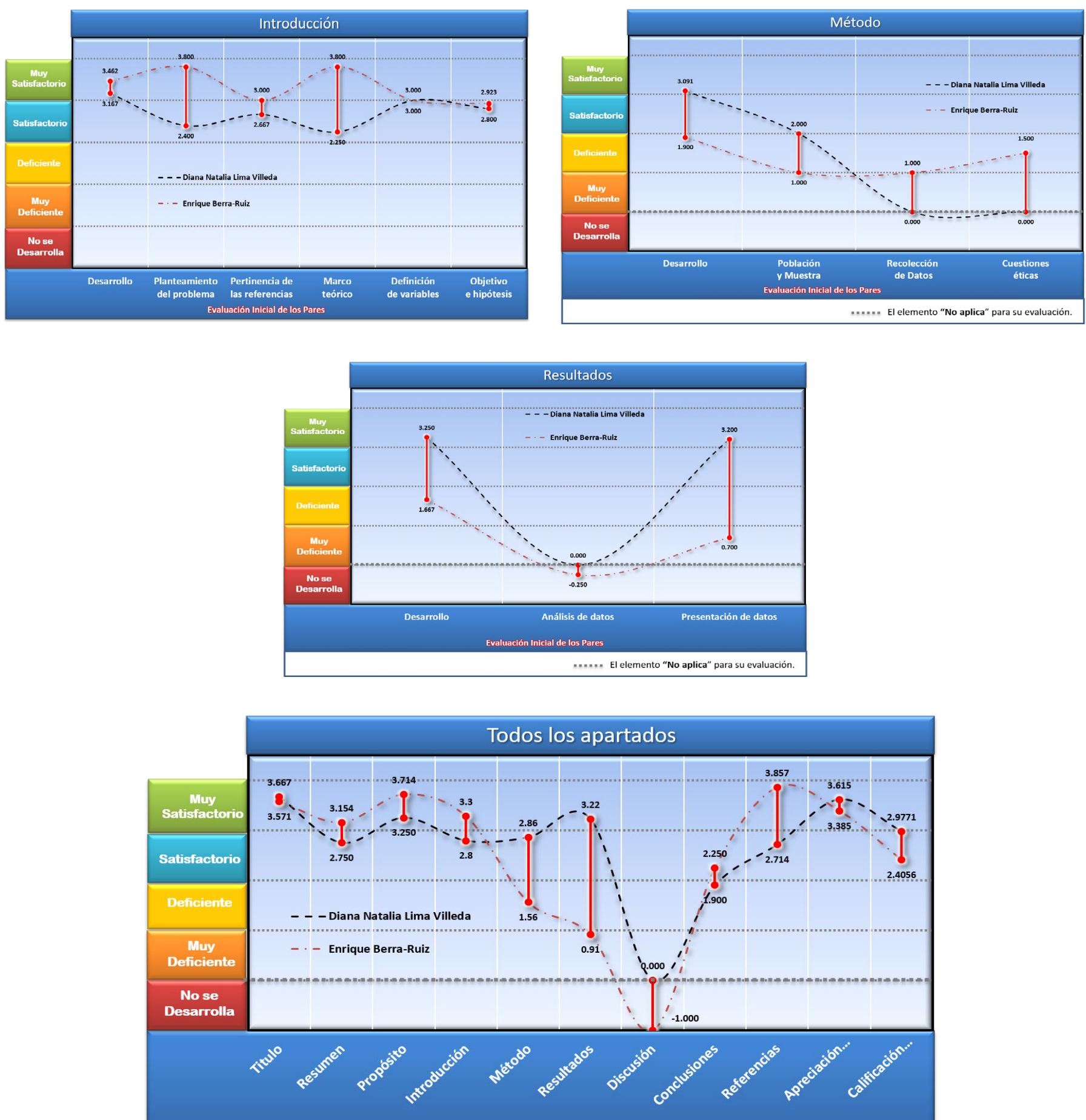

Evaluación Inicial de los Pares

" " +.". " El elemento “No aplica” para su evaluación. 


\section{Índice de Concordancia}

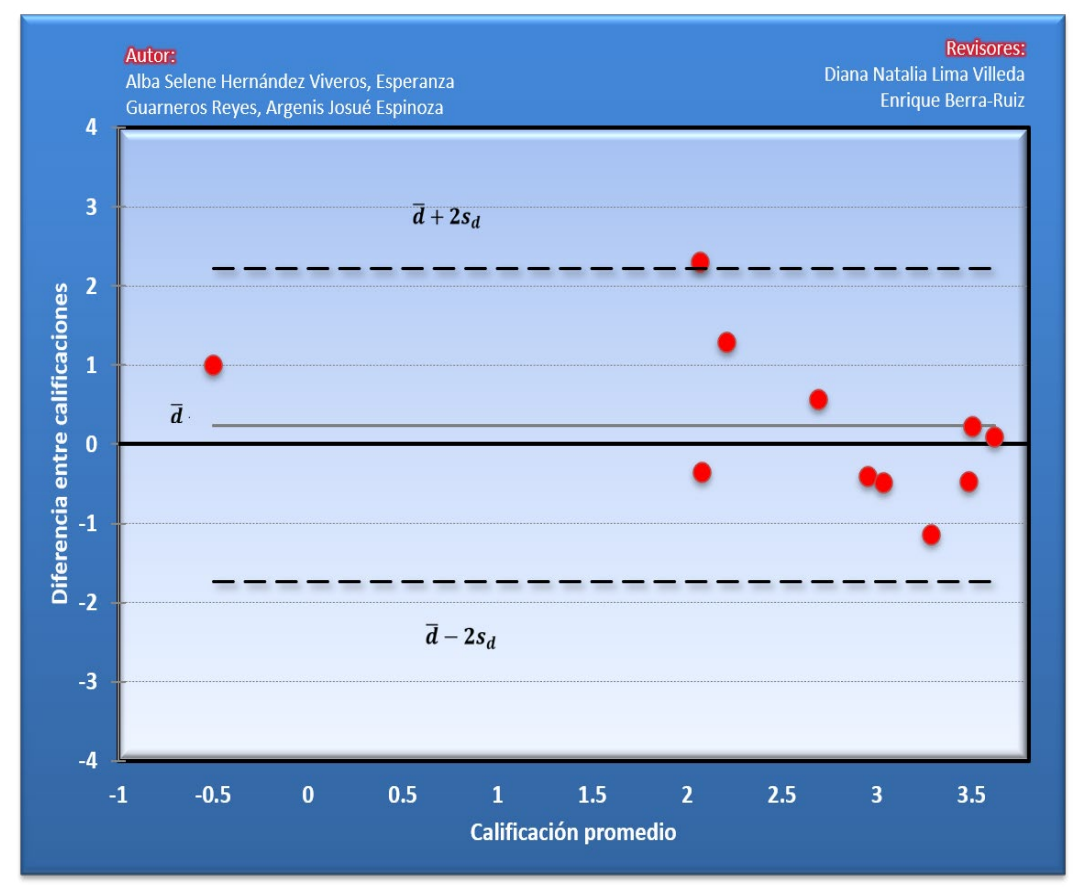

Índice de Acuerdo

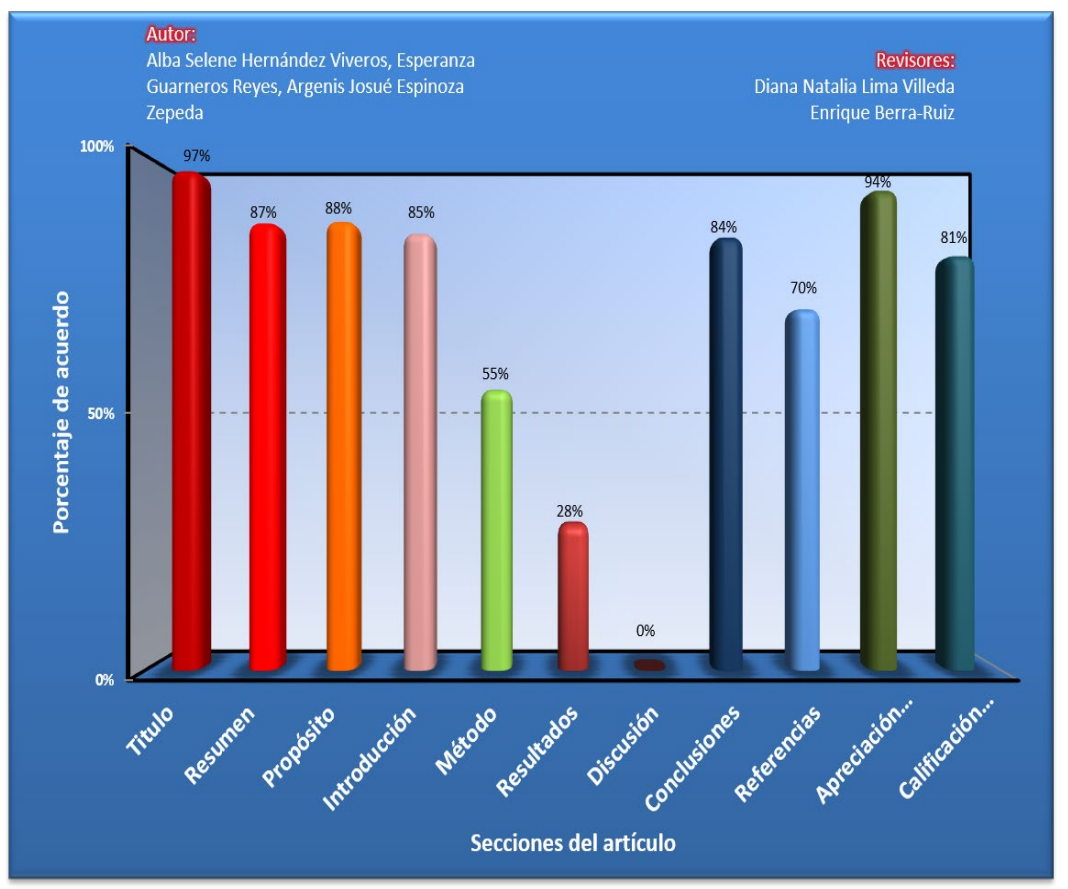




\begin{tabular}{|l|}
\hline \multicolumn{1}{|c|}{ Revisor 1 } \\
\hline \multicolumn{1}{|c|}{ Enrique Berra-Ruiz } \\
\hline \multicolumn{2}{|c|}{ Título/Aur } \\
\hline $\begin{array}{l}\text { El título aborda el principal objetivo del desarrollo del } \\
\text { contenido pero no se aborda el tema central del proceso } \\
\text { de enseñanza que enmarca y se centra más en el empleo } \\
\text { de la tecnología para generar nuevas espacios de aprendi- } \\
\text { zaje, independientemente el tema a abordar. }\end{array}$
\end{tabular}

Revisor 2

Diana Natalia Lima Villeda

\section{Resumen}

El título en general cubre satisfactoriamente con los criterios de evaluación de la revista, yo sugiero que en el mismo se incluya Desarrollo o propuesta de un simulador digital en moodle... dado que así, desde el mismo título se puede advertir al lector que lo que encontrará es la descripción de simulador, y que no se encontrarán datos por su uso.

El resumen entiendo debe ajustarse a 150 palabras, sobre pasó el número de ellas y sé que puede ser dificil dado todo el trabajo que se mostrará de manera extensa, sin embargo, creo que podría sustituirse "SUAyED Iztacala de la carrera de Psicología" por estudiantes "universitarios en modalidad en línea" dado que las características particulares de ese sistema educativo no dan cuenta de cómo los estudiantes de formación profesional en modalidades a distancia mediadas por tecnología se pueden beneficiar, no sólo los de esa población y en términos de número de palabras se ahorran al menos dos y algunas más por la redacción. En el sentido de abreviaturas pues el uso de SUAyED también perjudica, se usa y no es de domino común.

\section{Próposito del Estudio}

El propósito del proyecto es claro y pertinente y deriva una temática a desarrollar en donde se pueda aplicar.
Entiendo que, por la naturaleza del estudio y por las características que pide la revista para la publicación de éste tipo de contribuciones, no se incluye de manera tan clara una pregunta de investigación o hipótesis, aunado a que el estudio de la autora es la descripción de cómo se desarrolló el simulador, sin embargo, (aunque puse no aplica por las razones anteriores) considero que sí es importante que se establezca una pregunta, justo porque al final, cuando se refiere a las conclusiones, se puede abordar parte de la justificación de la necesidad de incluir este tipo de herramienta educativa en tales escenarios formativos y cómo el desarrollo de tales innovaciones tienen impacto directo en la formación universitaria. Es importante incluirlo. 
Innovación y desarrollo tecnológico | Simulación digital en Moodle ... Hernández-Viveros, Guarneros-Reyes ę Espinoza-Zepeda

\section{Revisor 1}

Revisor 2

\section{Introducción}

Se presenta el desarrollo de una herramienta tecnológica, pero al ser un desarrollo requiere también de una evaluación que si bien se establecen en los objetivos, no se plantean las variables a evaluar de forma clara y operacionalmente.
Considero que este tipo de trabajos es fundamental, sin embargo, para poder leerlo en términos de cómo la innovación es necesaria, considero que la justificación (en SUAyED sólo usan documentos o textos que no permiten o no favorecen la formación práctica, pero de alguna forma sí conviene sustentar esa afirmación, sobre todo porque la descripción es particular a esa población, no a las formaciones que se imparten en esa modalidad, además, de que no es explícito el marco teórico, y tampoco una pregunta de investigación, la pregunta que mejor se ciñe a éste estudio pero es cita (Guarneros, 2017) creo que da una pauta importante para la autora de éste documento de cómo elaborarla y darle sentido (teoría, justificación, literatura) a la luz de todo su trabajo que es muy importante.

\section{Método}

Si bien no es un estudio de investigación, el desarrollo de la tecnología marca una metodología a seguir así como un proceso de evaluación los cuáles sólo se mencionan y no se describen a detalle.
Considero que proponer un simulador digital como herramienta de aprendizaje en ambientes profesionales no aplican muchos de los elementos valorados, sin embargo, considero que sí se puede explicitar aun más la población dado que, es una innovación que puede ser utilizada en éstos ambientes formativos mediados por tecnología y que requieren el desarrollo de competencias más que el uso teórico de las nociones disciplinares.

\section{Resultados}

Se describen la funcionalidad y beneficios del desarrollo pero no se presentan resultados de acuerdo a los objetivos planteados.
Considero que las secciones rango de aplicación y funcionamiento son las más cercanas a la descripción de resultados, ahí aun se señala citas, como argumentando la pertinencia y relevancia de la innovación, cuando pueden señalarse las ventajas y bondades de aplicarse dado el diseño que siguieron y la herramienta moodle.

\section{Discusión}

No se desarrolla un apartado de discusión

al no tener resultados empíricos, considero que no aplica la sección de discusión. 


\begin{tabular}{|c|c|}
\hline Revisor 1 & Revisor 2 \\
\hline \multicolumn{2}{|c|}{ Conclusiones } \\
\hline $\begin{array}{l}\text { Las conclusiones analizan las fortalezas y empleabili- } \\
\text { dad del producto obtenido para una población en par- } \\
\text { ticular pero no aborda todos los objetivos a desarrollar. }\end{array}$ & $\begin{array}{l}\text { Considero que en las conclusiones se pueden señalar } \\
\text { cómo puede valorarse la efectividad del simulador en } \\
\text { términos de aprendizaje o revisión o ingreso de los es- } \\
\text { tudiantes a la plataforma o incluso, comentarios o vi- } \\
\text { vencias de los estudiantes con éste simulador, pero no } \\
\text { se señala nada al respecto. O al menos señalar qué re- } \\
\text { sultados esperan en términos de aprendizaje al imple- } \\
\text { mentar el simulador, no sólo repetir el discurso revisa- } \\
\text { do en la introducción. }\end{array}$ \\
\hline \multicolumn{2}{|c|}{ Referencias } \\
\hline El apartado de referencias es apropiado & $\begin{array}{l}\text { Hay referencias que no están citadas, tal vez es cómo } \\
\text { lo citan por ejemplo go animate o Hung. El formato } \\
\text { para las revistas no se sigue (con cursivas sin pp para } \\
\text { las páginas, etc.). son } 16 \text { referencias de las cuales } 10 \text { son } \\
\text { anteriores a } 2015 \text {. }\end{array}$ \\
\hline
\end{tabular}

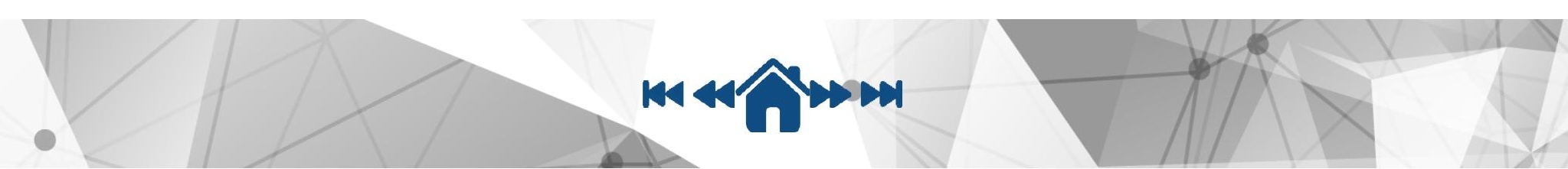


Innovación y desarrollo tecnológico | Simulación digital en Moodle ... Hernández-Viveros, Guarneros-Reyes \&Z Espinoza-Zepeda

\section{Historia del Proceso}

\section{EDITORIAL}

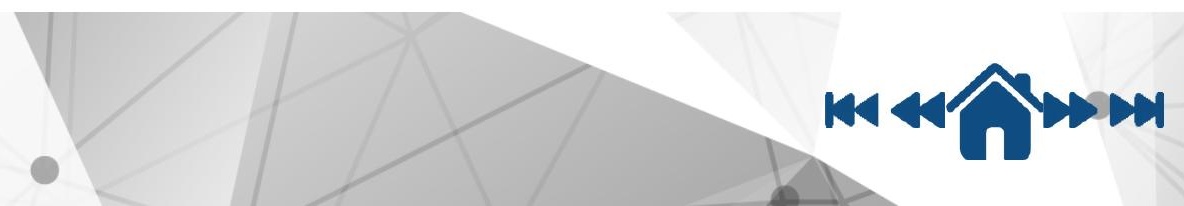

